\title{
KINEMATICAL FEATURES OF THE MAIN BULGE IN A MULTI-COMPONENT GALACTIC MODEL
}

\section{RAFAEL CUBARSI and MANUEL HERNANDEZ-PAJARES \\ Departament de Matematica Aplicada i Telematica, UPC P.O. Box 30002, E-08080 BARCELONA, Spain}

The present state of the Galaxy can be idealized as a stellar system with several components: inner bulge, main bulge, thin disk, thick disk, stellar halo, corona, etc. In the regions where the diffusion phenomena are negligible, the dynamics can be determined from a conservative dynamic system, according to the superposition principle with a common potential. Here we describe the kinematical behavior of the main bulge, thin and thick disks, and stellar halo, depending on the cylindrical galactocentric coordinates $r$ and $z$, under axisymmetric conditions. The angular dependence is treated in the work by Sanz-Subirana \& Juan-Zornoza (1992).

Our model is based in the following two hypotheses: (a) The stars are moving under a gravitational potential $U(t, \mathbf{r})$ and, since the model is not applied to the inner bulge, gas or corona, the collisionless Boltzmann equation is satisfied for all the components. (b) Accordin' to Oort's approximation, the velocity distribution of one stellar component is idealized by a Gaussian function of the peculiar velocities.

For a time-depending axisymmetric model this situation leads us to Stäckel potentials (Sala, 1990). However, in the case of superposition of several stellar components, the more general potential function has the following form (Cubarsí, 1990)

$$
U(\mathrm{t}, \mathbf{r})=A(t)\left(r^{2}+z^{2}\right)+k^{-1} U_{1}\left(k^{-1}\left(r^{2}+z^{2}\right)\right)+\left(r^{2}+z^{2}\right)^{-1} U_{2}\left(z^{2} r^{-2}\right)
$$

where $U_{1}, U_{2}, k(t)$ and $A$ are arbitrary functions of the specified arguments.

For each stellar component the mean velocity field and the velocity distribution are completely determined. In particular, the rotational mean velocity and the diagonal central moments for the $i$-th component are

$$
\begin{array}{ll}
\Theta_{0}{ }^{(i)}=-\Omega_{i} r\left(1+a_{i} r^{2}+b_{i} z^{2}\right)^{-1} ; & \mu_{r r}{ }^{(i)}=k_{i}^{-1}\left(1+b_{i} r^{2}\right)\left(1+b_{i}\left(r^{2}+z^{2}\right)\right)^{-1} \\
\mu_{\theta \theta}{ }^{(i)}=k_{i}^{-1}\left(1+a r_{i}^{2}+b_{i} z^{2}\right)^{-1} ; & \mu_{z z}{ }^{(i)}=k_{i}^{-1}\left(1+b_{i} z^{2}\right)\left(1+b_{i}\left(r^{2}+z^{2}\right)\right)^{-1}
\end{array}
$$

being $a_{i}, b_{i}, k_{i}$, and $\Omega_{i}$ specific parameters of the components.

The mass distribution of one component is modeled by the the potential and by the particular kinematical parameters of the component. Thus we have,

$$
N(t, \mathbf{r}) \propto \frac{\exp \left(\frac{1}{2} k_{i} \Omega_{i}^{2} r^{2}\left(1+a_{i} r^{2}+b_{i} z^{2}\right)^{-1}-k_{i}\left(U(\mathrm{t}, \mathbf{r})-A(t)\left(r^{2}+z^{2}\right)\right)-k_{i} b_{i} U_{2}\left(z^{2} r^{-2}\right)\right)}{\left(k_{i}^{3}\left(1+a_{i} r^{2}+b_{i} z^{2}\right)\left(1+b_{i}\left(r^{2}+z^{2}\right)\right)\right)^{1 / 2}}
$$

where $k_{i}=\alpha_{i} k(t)$, with $\alpha_{i}$ constant.

The imprecise information about numerical values of the Galactic potential, stellar density or velocity distribution for different regions of the Galaxy, in particular for the bulge, moves us to use an schematic version of the potential with $U_{1}=c k^{-1}\left(r^{2}+z^{2}\right)$ and $U_{2}=q+s z^{2} r^{-2} \quad(q, s$ and $c$ constants). The resulting potential has one term corresponding to an harmonic function, significant at large distances of the Galactic center, 
and another term generating a short-range force. The corresponding stellar density is dominated by the exponential factor with three differentiated contributions: (1) A term due to the rotation of the stellar component. If the component is fast rotating, like the thin disk and the bulge, it can be more important than the other terms. (2) A term due to the harmonic part of the potential and to the specific kinematics of the component. If $c>0$, this term fixes the scale length of the Galactic components. (3) A term depending on the potential and also on the particular kinematics of the component, which is significant near the center. If $q<0$, the short-range force is attractive and this term produces an exponential increasing of the mass density towards the center.

According to this model, we can compare the kinematics and mass distribution of the main bulge, disk and stellar halo. But since there are not definitive data about the potential, we focus in the Galactic plane. For the parameters of the potential, and in order to fix the characterristic length of the thick disk in $4.5 \mathrm{kpc}$ (Kruit, 1987), we take $c k^{-2}=850$ $\mathrm{kpc}^{-2}(\mathrm{~km} / \mathrm{s})^{2}$, consistent with the accepted local potential values (Ninkovic, 1990). In order to obtain decreassing mass density functions in $r$ for the bulge, we take $q=-3000 \mathrm{kpc}^{2}(\mathrm{~km} / \mathrm{s})^{2}$ (case $a$ ), otherwise $q$ can vanish (case $b$ ) since this value only modifies the stellar density within $r<0.75 \mathrm{kpc}$. The kinematical features of the thin disk, thick disk and stellar halo at the solar neighborhood (Gilmore \& Wyse, 1987; Sandage \& Fouts, 1987; Cubarsí, 1992; Cubarsí et al. 1992) are shown in the table bellow. For the main bulge we take the velocity dispersions similarly to the stellar halo (Rich, 1992), in particular the first component. In the table, their values are given at $0.5 \mathrm{kpc}$ and the tentative rotational velocity has a maximum of $250 \mathrm{~km} / \mathrm{s}$ at $0.3 \mathrm{kpc}$. The comparative parameters are: $h$ the characteristic length of the component, $R$ the distance which satisfies $N(R)=N(h) e^{-1}(N$ is the normalized stellar density), $H$ the distance with maximum stellar density, $d$ the distance with maximum rotational velocity, and $V$ the maximum rotational velocity value. The distances are given in $\mathrm{kpc}$ and the velocities in $\mathrm{km} / \mathrm{s}$. This method has been used in the synthetic approach in order to detect bulge stars in the solar neighborhood (Hernández-Pajares et al. 1992).

\begin{tabular}{|lllllll|}
\hline \multicolumn{1}{l}{} & $\left(\sigma_{r}: \sigma_{\theta}: \sigma_{z}\right)$ & $h$ & $R$ & $H$ & $d$ & $V$ \\
\cline { 2 - 7 } bulge & $(130: 65: 65)$ & 2 & 6 & $0(a) / 0.75(b)$ & 0.3 & 250 \\
thin-d & $(28: 17: 17)$ & $4.5-6.5$ & $4-7$ & 5.5 & 6.5 & 230 \\
thick-d & $(70: 50: 45)$ & 4.5 & 8 & 0 & 9 & 185 \\
halo & $(130: 105: 85)$ & 4.5 & 10 & 0 & 11.5 & 140 \\
\hline
\end{tabular}

\section{REFERENCES}

Cubarsí, R. (1990) Astron. J. 99, 1558.

Cubarsí, R. (1992) Astron. J. 103, 1608.

Cubarsí et al. (1992) Astronomy from Large Databases II. ESO. Haguenau.

Gilmore, G.; Wise, R.F.G. (1987) The Galaxy. Reidel:Dordrecht, p247.

Hernández-Pajares, M. et al.(1992) This Symposium.

Kruit, P.C. van der (1987) The Galaxy. Reidel:Dordrecht, p27.

Ninkovic, S. (1990) Bull. Astron. Inst. Czechosl. 41, 236.

Rich, R.M. (1992) The Stellar Population of Galaxies. Reidel:Dordrecht, p29.

Sala, F. (1990) Astron. Astrophys. 235, 85.

Sandage, A.; Fouts, G. (1987) Astron. J. 93, 74.

Sanz-Suhirana, J.; Juan-7ornoza, J.M. (1992) This Symposium.

Ackriswledperrients: Work :upported by the spanish grant PB90-(0478 from DGICIT. 